\title{
Migraine Associated Vertigo
}

\author{
Yoon-Hee Cha, M.D., Robert W. Baloh, M.D. \\ University of California, Los Angeles, California, USA
}

\begin{abstract}
The interrelations of migraine and vertigo are complex, eluding a simple localization either centrally or peripherally. Spontaneous episodic vertigo, benign paroxysmal positional vertigo, and Meniere's disease all occur more frequently in patients with migraine than in those without. Family studies support a hereditary predisposition to migraine associated vertigo. In this review, we discuss definitions, epidemiology, associated syndromes, neurootological abnormalities, genetics and treatment for patients with migraine and vertigo.
\end{abstract}

J Clin Neurol 3(3):121-126, 2007

Key Words : Migraine, Dizziness, Vertigo, BPPV, Benign recurrent vertigo, Meniere's disease, Motion sickness

\section{INTRODUCTION}

The prevalence of all forms dizziness has been shown to be higher in people with migraine than in the general population with some indicating true damage to the inner ear and others implicating problems with central vestibular processing. The aim of this review is to elucidate some of those associations with specific emphasis on migraine associated vertigo.

\section{PROPOSED DEFINITIONS}

The 2004 International Headache Society criteria for migraine did not recognize migrainous vertigo as a headache subtype ${ }^{1}$ but several prominent investigators have proposed working criteria. Neuhauser et al. have required at least two episodes of at least moderately severe vertigo concurrent with migrainous features which can include headache, photophobia, phonophobia, or visual aura. ${ }^{2}$ Their definition of vestibular vertigo includes: 1] rotational vertigo 2] positional vertigo 3] dizziness with nausea and either oscillopsia or imbalance. Crevits proposed similar criteria which includes at least two attacks of vertigo accompanied by migraine headache or visual aura. ${ }^{3}$ However, there are no uniformly agreed upon definitions of migrainous vertigo, or even the terminology. In this review, we will refer to episodic vertigo spells that occur concurrently with other migraine features as "migrainous vertigo," and episodic vertigo, which occurs in a patient with migraine, as "migraine-associated vertigo". The application of the term, "migraine-associated vertigo," does not require that the vertigo and headache occur simultaneously.

In order to capture the true relevance of vertigo in migraine, we will focus on migraine-associated vertigo. Several studies have shown that vertigo attacks and migraine headaches often do not occur simultaneously. Brantberg and Baloh performed a study of 40 migraine patients and 40 relatives with recurrent attacks of

Received : August 27, 2007 / Address for correspondence : Robert W. Baloh, M.D.

710 Westwood Plaza, Box 951769 Los Angeles, CA, USA 90095

E-mail: rwbaloh@ucla.edu

${ }^{*} \mathrm{NIH}$ grant P50 DC 05224 from the NIDCD, NIH grant 5 U54 RR019482, and the Clinical Research Training Fellowship from the American Academy of Neurology. 
rotational vertigo in the absence of hearing loss and found that only half of the patients reported ever experiencing headache along with vertigo. ${ }^{4}$ This was consistent with Kayan and Hood's findings that 36\% of patients with vertigo and migraine experienced vertigo in headache-free periods. ${ }^{5}$ In a study by Cutrer and Baloh of 91 patients with migraine and vertigo, only 5 patients reported a consistent relationship between the headaches and vertigo. ${ }^{6}$ Oh et al. studied 24 patients with recurrent vertigo and their family members and found a migraine prevalence of $83 \%$ in probands and $70 \%(31 / 44)$ in relatives who also experienced recurrent vertigo. Only $60 \%$ (12/20) of probands had had some of their migraine headaches along with vertigo attacks. ${ }^{7}$ When migraine and recurrent vertigo are seen in the same patient, the temporal concurrence of these symptoms is somewhere between $30-70 \%{ }^{8-9}$

\section{EPIDEMIOLOGY}

Epidemiological studies have shown a steady prevalence of migraine in the United States when care is taken to follow strict International Headache Society criteria. The rates range from $16-18 \%$ for women and $5-6 \%$ for men. ${ }^{10,11}$ The prevalence of migraine was shown to be significantly higher at $38 \%$ in a prospective study of 200 patients recruited from a dizziness clinic, with migrainous vertigo specifically seen at a prevalence of $7 \% .^{2}$ Interestingly, in this study, the rate of migraine in the control population of orthopedic patients was $24 \%$, somewhat higher than population averages. In a German population survey, ${ }^{8} 1212$ of 4077 (30\%) participants consenting to a telephone interview reported having experienced moderate or severe dizziness/vertigo in their lifetimes. Of the 1003 participants who eventually completed all the required interviews, $243(24 \%)$ reported specifically experiencing vestibular vertigo. Of these 243 , $40 \%$ experienced spontaneous rotational vertigo, $43 \%$ positional vertigo and $17 \%$ episodic nausea with oscillopsia or imbalance. ${ }^{8}$ A follow-up analysis of this same data sample showed that $3.2 \%$ of participants had a history of migraine-associated vertigo and $0.9 \%$ met their criteria for migrainous vertigo. ${ }^{12}$ Twenty seven percent of the 200 migraine patients in Kayan and Hood's study experienced vertigo. ${ }^{5}$ If applied to a population prevalence of migraine of $18 \%$ in women and $6 \%$ in men, this would mean that about $4.5 \%$ of women and $1.5 \%$ of men would be expected to experience migraine-associated vertigo, which are not too dissimilar to the German study's finding.

\section{MIGRAINE ASSOCIATED VERTIGO SYNDROMES}

\section{Benign recurrent vertigo of childhood}

Basser first reported on a syndrome of paroxysmal vertigo affecting children generally before the age of $8 .^{13}$ Attacks were brief lasting seconds to minutes and had a benign prognosis other than being associated with a caloric paresis. Fenichel later showed the connection between the disorder and migraine headaches. ${ }^{14}$ Subsequent long-term follow up studies have confirmed the association with migraine, showing both a high rate of personal and family history of migraine in children with benign paroxysmal vertigo. ${ }^{15-18}$ Additionally, most of these children also suffered from motion sickness. ${ }^{16-18}$

\section{Meniere's disease}

The literature on the association of migraine and Meniere's disease is necessarily confusing as much has been printed before the acceptance of clear criteria for either disorder and even recent papers do not indicate whether the diagnosis of Meniere's disease is based on definite, probable or possible features. It is no surprise, therefore, that the prevalence of migraine in Meniere's disease has ranged considerably (22$76 \%$ ), ${ }^{19-22}$ though most studies do indicate a higher than population baseline of migraine in Meniere's patients. The association is complex, however, because migraine can mimic the symptoms of Meniere's disease and migraine may even damage the inner ear 
causing a delayed endolymphatic hydrops. ${ }^{23}$

We observed that certain clinical features are more prevalent in patients with migraine and Meniere's disease versus those with Meniere's disease alone. In our study, patients with migraine and Meniere's disease had an earlier age of onset, a higher rate of concurrent bilateral hearing loss and greater family history of migraine headaches and episodic vertigo than patients without migraine. ${ }^{24}$ Familial forms of Meniere's disease have been presented throughout the years and most of these families also show high rates of migraine and episodic vertigo without hearing loss ${ }^{25-28}$ indicating that a "migraine-Meniere's" syndrome is likely genetically distinct from Meniere's disease alone.

\section{Basilar migraine}

Though the current IHS criteria do not recognize recurrent vertigo as a migraine equivalent symptom, vertigo is included as one of the many symptoms of basilar migraine. The strict definition of basilar migraine requires an aura of bilateral visual field abnormalities along with at least two symptoms localizable to the brainstem in association with a migraine headache. ${ }^{1}$ Vertigo is one of the most common symptoms in basilar migraine. ${ }^{29}$ However, the majority of patients with migraine and vertigo do not qualify for a diagnosis of basilar migraine as the vertigo attacks are often separate from the headaches and the duration of the vertigo often does not fall within the restrictions for aura (5-60 min). ${ }^{2,4,9,30}$

\section{BPPV}

The pathophysiology of benign paroxysmal positional vertigo (BPPV) relates to degenerating otoconia loosened in the labyrinth. Otolith movement causing endolymphatic fluid flow creates a brief spell of dizziness, which patients usually describe as true rotatory vertigo, nausea, light-headedness or imbalance. BPPV is associated with brief torsional geotropicbeating nystagmus in the head-hanging position ${ }^{31}$ and is successfully treated with the particle repositioning maneuver. $^{32}$ This should be distinguished from positional worsening of symptoms in other forms of vertigo, including migrainous vertigo. ${ }^{33}$ Several studies have shown high prevalence rates of migraine in idiopathic BPPV. A retrospective study by Uneri in 476 patients with posterior canal variant BPPV revealed a history of migraine meeting IHS criteria in $54.8 \%$ of patients; $67.4 \%$ of these 476 patients reported a history of motion sensitivity, both rates that are much higher than population baseline. ${ }^{34}$ Ishiyama et al. studied 247 patients with BPPV and found that a history of migraine was 3 times more common in patients with idiopathic BPPV than in those who developed it after head trauma or surgery. ${ }^{35}$ von Brevern et al. performed a population based study of patients with dizziness/ vertigo using historical criteria for BPPV (at least 5 attacks of dizziness triggered by typical head position changes lasting less than 1 minute). The odds ratio for migraine in their BPPV group versus control was 7.5. ${ }^{36}$ These studies indicate that migraine is a risk factor for BPPV. Though the mechanism is unknown, it may relate to small degrees of ischemic damage to the otoliths, perhaps through vasospasm. ${ }^{23,37}$

\section{NEUROTOLOGICAL FEATURES}

\section{Electronystagmographic (ENG) abnormalities}

Several studies have observed ENG abnormalities in migraine patients though most studies have shown that the range of degree of caloric paresis is generally mild. Cutrer and Baloh reviewed vestibular testing abnormalities in 91 patients with migraine associated dizziness and found unilateral vestibular paresis in $20.9 \%$ of the patients. ${ }^{6}$ Smaller studies have reported similar rates. $^{38-40}$ The role of symptomatic vertigo is unclear, however, because caloric abnormalities have also been observed in migraine patients without a history of vestibular symptoms. ${ }^{39}$ Given the vagaries of caloric testing, one must be cautious in ascribing mild degrees of paresis to any particular disorder. Subtle abnormalities such as spontaneous nystagmus, directional preponderance, and static positional nystagmus 
have also been observed in migraine patients. ${ }^{6}$ Though these are generally non-localizing findings, they may indicate an imbalance in the vestibular pathways.

Ocular-motor features during acute migrainous vertigo attacks were evaluated by von Brevern et al. in 20 patients with migraine. Ten patients had features of central nystagmus, 3 peripheral nystagmus and 7 which were indeterminant. All variations of upbeat, downbeat, horizontal and torsional nystagmus were observed. ${ }^{41}$ Kayan and Hood studied electronystagmographic recordings in 80 migraine patients in between headaches and found central abnormalities in 11, peripheral in 6 and indeterminant findings in $14 .^{5}$ These studies highlight the difficulty in ascribing a pure central or peripheral localization to migraine associated vertigo.

\section{GENETICS}

Episodic vertigo occurs in association with rare genetic syndromes, some of which have known genes. Several of the episodic ataxias (EA 1,2,3,4) have at least some reported features of vertigo ${ }^{42-45}$ but only episodic ataxia type 2 (EA2) has a strong association with migraine. About $50 \%$ of EA2 patients experience migraine headaches, which is no surprise as it is allelic with familial hemiplegic migraine (FHM) type $1 .^{43}$ There is also growing evidence that episodic vertigo in association with migraine also has a strong genetic component. In Oh's report on familial benign recurrent vertigo of 24 probands, the prevalence of spontaneous attacks of vertigo in relatives was $40 \%$ as opposed to $2 \%$ in unrelated spouses and was also correlated with a high prevalence of migraine $(46 \%)$ in family members. In 22 of the cases, there was more than one generation affected making an autosomal dominant pattern of inheritance the most likely. ${ }^{7}$ The only genetic association study to date was on 20 families with episodic vertigo in which at least 3 family members were affected. This study found an association with $22 \mathrm{q} 12 .^{46}$ The majority of these patients and family members also had migraine headaches.

\section{TREATMENT}

When patients with migraine associated vertigo present with clear features of BPPV or Meniere's disease, they should be treated with standard treatment for those disorders (particle repositioning maneuver for BPPV, diuretics, salt restriction and possible surgical intervention for Meniere's disease). Meniere's attacks associated with prominent headache can also be treated with calcium channel blockers.

The management of acute spells depends largely on the duration and severity. Severe spells that last longer than 20 minutes can be treated pharmacologically. If the patient is able to swallow a pill, then oral meclizine, promethazine or prochloperazine can be used. We prefer promethazine for its efficacy and because it can be substituted 1:1 in the suppository form, which can be used if nausea from the vertigo is severe. All antiemetics are potentially sedating so patients should be adequately warned not to drive after taking these medications. Many patients find that a good nap or good night's sleep will break a vertigo attack and welcome the sedating effects. Benzodiazepines are also effective vestibular suppressants but have the potential for tolerance and dependency and should be used with caution.

As is the true for all episodic disorders, the mainstay of preventative treatment is to avoid triggers. Many patients report triggers for vertigo that are very similar to triggers for typical migraine headaches. These include stress, lack of sleep, dietary fluctuations, fluctuations in caffeine intake, certain kinds of foods, weather changes and menstrual cycles. Controlled trials of medications for idiopathic recurrent vertigo are lacking but anecdotal reports of the efficacy of acetazolamide have lead to the more widespread use of this medication. We find it helpful for patients who have clear attacks of rotatory vertigo. The rationale for trying acetazolamide, a carbonic anhydrase inhibitor was based on the efficacy of this drug in reducing recurrent vertigo in patients with EA2. ${ }^{47}$ The action of acetazolamide in the brain that renders its efficacy is still unknown, but $\mathrm{P}^{31} \mathrm{MRS}$ have shown that treatment 
lowers the $\mathrm{pH}$ of the extracellular space in patients with episodic ataxia. ${ }^{48}$ An acidic extracellular environment leads to decreased neuronal excitability, whereas an alkaline environment is associated with increased excitability. The main side effects of acetazolamide include paresthesias, dehydration, lethargy and sometimes myalgias. Acetazolamide can also decrease potassium levels. In general, we start this medication at a low dose (125 mg a day) and only increase it by $125 \mathrm{mg}$ a week to a goal dose of $250 \mathrm{mg}$ twice daily. We encourage patients to drink generous amounts of citrus juices to avoid the alkalinization of urine, which leads to the formation of kidney stones.

We also use verapamil in the extended release form, which works well for patients with recurrent vertigo and migraine headaches. A small case series of patients with recurrent vertigo and chronic headaches showed efficacy with typical anti-migraine medications including pizotifen, a calcium channel blocker, verapamil and propranolol. ${ }^{49}$ The longer acting form is associated with fewer drops in blood pressure and we are able to use this medication frequently in young women who generally have low blood pressure.

Selective serotonin reuptake inhibiting medications can be effective and are generally most useful in patients who have concurrent mood disorders. Chronic dizziness of any kind, particularly with unpredictable spells, are extremely anxiety provoking and can lead to a complex interaction of otogenic and psychogenic factors that can lead to prolonged disability. ${ }^{50}$ The use of SSRIs can help address the vertigo attacks as well as the adjustment reaction to the attacks.

\section{CONCLUSION}

Numerous studies support migraine as a clear risk factor for the development of both central and peripheral forms of vertigo. Like primary migraine headache, migraine-associated vertigo shows a strong genetic basis. Recognizing the various forms of migraineassociated vertigo is key to the efficient diagnosis, treatment, and education of this group of patients, from whom much can be learned about the complex interrelations of the brain and inner ear.

\section{REFERENCES}

1. Headache Classification Subcommittee of the International Headache Society. The International Classification of Headache Disorders 2nd edition. Cephalalgia 2004;24: 1-160.

2. Neuhauser H, Leopold M, von Brevern M, Arnold G, Lempert $\mathrm{T}$. The interrelations of migraine, vertigo, and migrainous vertigo. Neurology 2001;56:436-441.

3. Crevits L, Bosman T, Migraine-related vertigo: towards a distinctive entity. Clinical Neurology and Neurosurgery 2005;107:82-87.

4. Brantberg K, Trees N, Baloh RW. Migraine-associated vertigo. Acta Oto-Laryngologica 2005;125:276-279.

5. Kayan A, Hood JD. Neuro-otological manifestations of migraine. Brain 1984;107:1123-1142.

6. Cutrer F, Baloh RW. Migraine-associated dizziness. Headache 1992;32:300-304.

7. Oh A, Lee H, Jen JC, Corona S, Jacobson KM, Baloh RW. Familial Benign Recurrent Vertigo. Am Jrnl Med Genetics 2001;100:287-291.

8. Neuhauser HK, von Brevern M, Radtke A, Lezius F, Feldmann M, Ziese T, et al. Epidemiology of vestibular vertigo: A neurotologic survey of the general population. Neurology 2005;65:898-904.

9. Dieterich M, Brandt T, Episodic vertigo related to migraine (90 cases): vestibular migraine? J Neurol 1999;246: 883-892.

10. Lipton R, Scher AI, Kolodner K, Liberman J, Steiner TJ, Stewart WF. Migraine in the United States: Epidemiology and patterns of health care use. Neurology 2002;58:885894.

11. Lipton R, Bigal ME, Diamond M, Freitag F, Reed ML, Stewart WF. AMPP Advisory Group, Migraine prevalence, disease burden, and the need for preventive therapy. Neurology 2007;68:343-349.

12. Neuhauser H, Radtke A, von Brevern M, Feldmann M, Lezius F, Ziese T, et al. Migrainous vertigo: Prevalence and impact on quality of life. Neurology 2006;67:10281033.

13. Basser L. Benign paroxysmal vertigo of childhood (a variety of vestibular neuronitis). Brain 1964;87:141-152.

14. Fenichel G. Migraine as a cause of benign paroxysmal vertigo of childhood. J Pediatr 1967;71:114-115.

15. Lanzi G, Balottin U, Fazzi E, Tagliasacchi M, Manfrin M, Mira E. Benign paroxysmal vertigo of childgood: a long-term follow-up. Cephalalgia 1994;14:458-460. 
16. Abu-Arafeh I, Russell G. Paroxysmal vertigo as a migraine equivalent in children: a population based study. Cephalalgia 1995;15:22-25.

17. Marcelli V, Piazza F, Pisani F, Marciano E. Neurootological features of benign paroxysmal vertigo and benign paroxysmal positioning vertigo in children: a follow-up study. Brain Dev 2006;28:80-84.

18. Drigo P, Carli G, Laverda AM. Benign paroxysmal vertigo of childhood. Brain Dev 2001;23:38-41.

19. Radtke A, Lempert T, Gresty MA, Brookes GB, Bronstein AM, Neuhauser H. Migraine and Meniere's disease: Is there a link? Neurology 2002;59:1700-1704.

20. Hinchcliffe R. Headache and Meniere's Disease. Acta Oto-Laryngologica 1967;63:384-390.

21. Parker W. Meniere's Disease: Etiologic Considerations. Arch Otolaryngol Head Neck Surg 1995;121:377-382.

22. Rassekh C, Harker LA. The prevalence of migraine in Meniere's disease. Laryngoscope 1992;102:135-138.

23. Lee H, Lopez I, Ishiyama A, Baloh RW. Can Migraine Damage the Inner Ear? Arch Neurol 2000;57:1631-1634.

24. Cha YH, Brodsky J, Ishiyama G, Sabatti C, Baloh RW. The relevance of migraine in patients with Meniere's disease. Acta-otolaryngologica, in press, 2007.

25. Brown M. Meniere's syndrome. Arch Neurol Psychiatry 1941;46:561.

26. Brown M. The factor of heredity in labyrinthine deafness and paroxysmal vertigo (Meniere's syndrome). Ann Otol Rhino Laryngol 1949;58:665-70.

27. Bernstein J. Occurrence of episodic vertigo and hearing loss in families. Ann Otol Rhino Laryngol 1965;74:10111021.

28. Oliveira CA, Messias CI, Ferrari I. Occurrence of Familial Meniere's Syndrome and Migraine in Brasilia. Ann Otol Rhinol Laryngol 2002;111:229-236.

29. Sturzenegger MH, Meienberg O. Basilar artery migraine: a follow-up study of 82 cases. Headache 1985;25:408415.

30. Neuhauser H, Lempert T. Vertigo and dizziness related to migraine: a diagnostic challenge. Cephalalgia 2004;24: 83-91.

31. Baloh RW, Honrubia V, Jacobson KM., Benign positional vertigo: clinical and oculographic features in 240 cases. Neurology 1987;37:371-378.

32. Epley JM. The canalith repositioning procedure: for treatment of benign paroxysmal positional vertigo. Otolaryngol Head Neck Surg 1992;107:399-404.

33. von Brevern M, Radtke A, Clarke AH, Lempert T. Migrainous vertigo presenting as episodic positional vertigo. Neurology 2004;62:469-472.

34. Uneri, A. Migraine and benign paroxysmal positional vertigo: an outcome study of 476 patients. ENT-Ear, Nose \& Throat Journal 2004;83:814-815.
35. Ishiyama A, Jacobson KM, Baloh RW. Migraine and benign paroxysmal vertigo. Ann Otol Rhino Laryngol 2000;109:377-380.

36. von Brevern M, Radtke A, Lezius F, Feldmann M, Ziese $\mathrm{T}$, Lempert T, et al. Epidemiology of benign paroxysmal positional vertigo. J Neurol Neurosurg Psychiatry 2006. Epub Nov 29.

37. Viirre E, Baloh RW. Migraine as a cause of sudden hearing loss. Headache 1996;36:24-28.

38. Toglia JU, Thomas D, Kuritzky A. Common migraine and vestibular function: electronystagmographic study and pathogenesis. Ann Otol 1981;90:267-271.

39. Bir LS, Ardic FN, Kara CO, Akalin O, Pinar HS, Celiker A. Migraine patients with or without vertigo: comparison of clinical and electronystagmographic findings. J Otolaryngology 2003;32:234-238.

40. Kuritzky A, Toglia UJ, Thomas DO. Vestibular function in migraine. Headache 1981;21:110-112.

41. von Brevern M, Zeise D, Neuhauser H, Clarke AH, Lempert T. Acute migrainous vertigo: clinical and oculographic findings. Brain 2005;128:365-374.

42. Cader MZ, Steckley JL, Dyment DA, McLachlan RS, Ebers GC. A genome-wide screen and linkage mapping for a large pedigree with episodic ataxia. Neurology 2005;65:156-158.

43. Ophoff RA, Terwindt GM, Vergouwe MN, van Eijk R, Oefner PJ, Hoffman SMG, et al. Familial Hemiplegic Migraine and Episodic Ataxia Type-2 Are Caused by Mutations in the Ca2+ Channel Gene CACNL1A4. Cell 1996;87:543-552.

44. Browne DL, Gancher ST, Nutt JG, Brunt ER, Smith EA, Kramer P, et al. Episodic ataxia/myokymia syndrome is associated with point mutations in the human potassium channel gene, KCNA1. Nat Genet 1994;8:136-140.

45. Steckley JL, Ebers GC, Cader MZ, McLachlan RS. An autosomal dominant disorder with episodic ataxia, vertigo, and tinnitus. Neurology 2001:1499-1502.

46. Lee H, Jen JC, Wang H, Chen Z, Mamsa H, Sabatti C, et al. A genome-wide linkage scan of familial benign recurrent vertigo: linkage to $22 \mathrm{q} 12$ with evidence of heterogeneity. Hum. Mol. Genet 2006;15:251-258.

47. Zasorin N, Baloh RW, Meyers LB. Acetazolamideresponsive episodic ataxia syndrome. Neurology 1983; 33:1212-1214.

48. Sappey-Marinier D, Vighetto A, Peyron R, Broussolle E, Bonmartin A. Phosphorus and proton magnetic resonance spectroscopy in episodic ataxia type 2. Ann of Neurol 1999;46:256-259.

49. Waterston J. Chronic migrainous vertigo. Jrnl Clin NeurosC 2004;11:384-388.

50. Staab, JP, Ruckenstein MJ. Which Comes First? Psychogenic Dizziness versus Otogenic Anxiety. Laryngoscope 2003;113:1714-1718. 